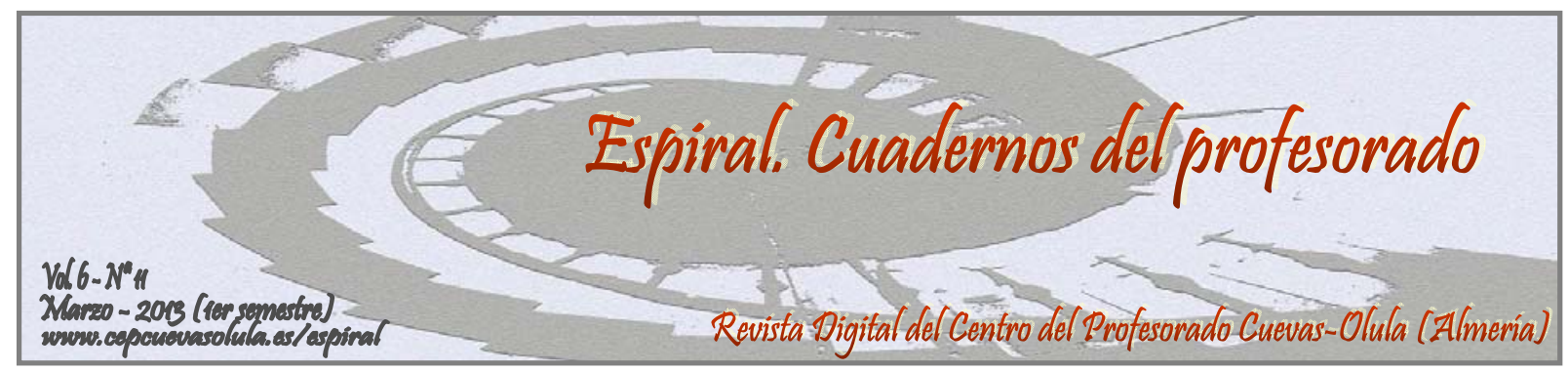

\title{
LA WEBQUEST EN LA ENSEÑANZA DEL BALONMANO
}

WEBQUEST IN TEACHING OF HANDBALL

\section{Manuel Gómez-López}

Facultad de Ciencias del Deporte, Universidad de Murcia, España

RESUMEN: La presente experiencia resalta el fomento de las Tecnologías de la Información y la Comunicación (TIC) como recurso del profesor, en concreto a través de la elaboración de una WebQuest. Esta herramienta sirvió de apoyo para la enseñanza de los contenidos relativos a la especialidad deportiva de Balonmano en las clases de Educación Física de Educación Secundaria Obligatoria y Bachillerato en dos centros diferentes de la Región de Murcia. A partir de los resultados positivos que hemos alcanzado, se puede afirmar que este tipo de herramientas incentiva el aprendizaje del alumno y la aplicación de las TIC en las aulas.

Palabras clave: WebQuest, competencias, TIC

ABSTRACT: This experience highlights the promotion of Information Technology and Communication (ICT) as a teacher resource, particularly through the development of a WebQuest. This tool was used to support the teaching of content related to the sport of Handball specialty classes in Physical Education Secondary School Education in two different centers of the Region of Murcia. Based on the positive results we have achieved, we can say that these tools encourages student learning and the application of ICT in the classroom.

Key words: WebQuest, skills, CIT

Gómez-López, M. (2013). La WebQuest en la enseñanza del Balonmano. Espiral. Cuadernos del Profesorado, 6(11), 28-35. Disponible en: http://www.cepcuevasolula.es/espiral.

Fecha de recepción: 1/06/2012

Fecha de aceptación: 14/11/2012
Enviar correspondencia a: mgomezlop@um.es

\section{1.- INTRODUCCIÓN}

Hoy día ya está aceptado que las Tecnologías de la Información y la Comunicación (TIC) son capaces de acercar personas, de facilitar el uso compartido de recursos, de permitir las realidades virtuales lo que está provocando un cambio notable en la forma de enseñar y de aprender. Las TIC facilitan nuevas visiones de la profesión profesor, la aparición de nuevos escenarios y de nuevas metodologías (Benito y Ovelar, 2005). Esto es debido a que el contexto educativo en el que nos encontramos envueltos, no puede mantenerse al margen de los progresos que alcanza la sociedad actual, la cual se caracteriza por 
el uso y el avance de las TIC. Debemos adaptarnos continuamente con espíritu crítico a estos cambios, buscando siempre nuevas formas de afrontar estos retos.

Gracias al uso de las TIC como profesores podemos conseguir competencias como la búsqueda y selección de información, el análisis crítico y la resolución de problemas, el trabajo en equipo, los idiomas, la capacidad de autoaprendizaje y de adaptación al cambio, la interdisciplinaridad o la iniciativa y la perseverancia (Mendaña y González, 2004; Huertas y Tenorio, 2006).

En este artículo describimos el proceso de elaboración y el contenido de una WebQuest, con la humilde intención de despertar nuevas ideas que enriquezcan las herramientas utilizadas por los profesores en las aulas y repercuta positivamente en el proceso de enseñanza-aprendizaje. Igualmente pretendemos que estas experiencias motiven al profesor en la utilización dentro de otros ámbitos del conocimiento, en los que exista la posibilidad de utilizar las TIC como herramienta didáctica.

\section{WEBQUEST: “DISEÑO DE UNA SESIÓN DE ENSEÑANZA DE LOS FUNDAMENTOS TÉCNICO-TÁCTICOS INDIVIDUALES EN BALONMANO”}

La WebQuest es una estrategia didáctica que se está popularizando en todo el mundo para integrar Internet y las nuevas tecnologías en el ámbito educativo y en especial en el universitario (Ruiz, Navarrete, Martínez y González, 2006), donde los profesores tenemos que innovar continuamente nuestras metodologías de enseñanza.

El creador de las WebQuest las define como una actividad de investigación en la que la información con la que interactúan los alumnos proviene total o parcialmente de recursos de Internet (Dodge, 1995). Según Yoder (1999), una WebQuest puede ser entendida como un tipo de unidad didáctica con vínculos a la web, que permite presentar al alumnado un escenario, una tarea, un problema a resolver o un proyecto para realizar. Adell (2004), por otro lado expone que una WebQuest es una actividad didáctica que propone una tarea factible y atractiva para los estudiantes y un proceso para realizarla durante el cual los alumnos harán cosas con información: analizar, sintetizar, comprender, transformar, crear, juzgar y valorar, crear nueva información, publicar, compartir, etc. Como señaló Area (2004), la WebQuest es la aplicación de estrategias de aprendizaje por descubrimiento guiado mediante trabajos desarrollados por el alumnado que utiliza los recursos de Internet.

Esta aplicación tecnológica tiene la finalidad de guiar al alumno en la búsqueda e investigación de una tarea propuesta, facilitándole a su vez las herramientas necesarias para ello, que en su mayoría, serán extraídas de Internet. De esta manera culminará la tarea con éxito a través de la búsqueda de información guiada y orientada a la investigación.

La tarea central de una WebQuest es una versión reducida de lo que las personas adultas hacen en el trabajo, fuera de los muros de la escuela (Starr, 2000).

Por tanto, las WebQuest son una estrategia de enseñanza-aprendizaje que se basan en principios constructivistas, en el aprendizaje por proyectos y en la indagación guiada a partir de recursos en su mayoría extraídos de Internet. Son proyectos didácticos, unidades de aprendizaje colaborativo que potencian la construcción del conocimiento (Castro, 2007).

Las WebQuest constituyen una estrategia didáctica en la que los alumnos son los constructores de su propio conocimiento, lo cual está en completa sintonía con la filosofía del EEES, en el que se persigue transmitir conocimientos básicos al tiempo que formarlo para que él pueda completar su formación en función de las necesidades que le van a ir surgiendo (Huertas y Tenorio, 2006).

Núñez (2011) expone que la WebQuest desarrolla las siguientes competencias en los alumnos: la búsqueda, procesamiento y aplicación de la información; la identificación y formulación de problemas; presentación, exposición y defensa de ideas; elaboración de comentarios, propuestas y evaluación; lectura y redacción y respeto a los aportes de otros autores.

La WebQuest a la que haremos referencia en este trabajo se diseñó para las asignaturas: Enseñanza del Balonmano y Fundamentos del Balonmano, y lleva por título:” Diseño de una sesión de enseñanza de los fundamentos técnico-tácticos individuales en Balonmano”. 
Esta herramienta se elaboró para ser utilizada en dos asignaturas de la especialidad de Balonmano. Por un lado para la asignatura de Enseñanza del Balonmano, incluida en el $2^{\circ}$ curso de la Licenciatura y para la asignatura de Fundamentos del Balonmano, correspondiente a $1^{\circ}$ curso de Grado en Ciencias de la Actividad Física y del Deporte. Al ser ambas asignaturas obligatorias en sus respectivos planes de estudios, se planteó la WebQuest teniendo en cuenta que los alumnos tenían adquiridos una serie de conocimientos previos necesarios para comprender y desarrollar el trabajo. El objetivo de la misma fue que los alumnos, fueran capaces de diseñar una sesión destinada a la iniciación en balonmano.

La página Web, en la que podemos encontrar dicha WebQuest es la siguiente: www.um.es/desarrollopsicomotor/wq/2010/wqma nuelabr2010/Introduccion.html

En cuanto al proceso seguido en su elaboración, hay que resaltar que a pesar de que también son válidas otras posibilidades, la siguiente propuesta de WebQuest se estructura a través del lenguaje HTML. El diseño de esta WebQuest se estructura en: introducción, tarea, procesos y recursos, evaluación y conclusión. Cada uno de estos apartados es explicado y ejemplificado con el contenido utilizado en la presente WebQuest.

Hay que resaltar que, como se ha indicado en la introducción, estas partes no están disociadas unas de otras sino que están interconectadas entre sí formando un auténtico sistema.

A continuación pasamos a detallar el diseño de esta WebQuest, atendiendo a las consideraciones de Díaz (2005), Fernández (2007) y Sosa (2008) (figura 1):

Introducción (figura 1): tiene dos objetivos fundamentales, por un lado orientar a los alumnos sobre lo que se van a encontrar y por otro incrementar su interés por la actividad. En este apartado se pretende hacer la actividad atractiva para el alumnado y darles nociones básicas sobre la tarea que deberán realizar. Este apartado se cerrará con el objetivo que se pretende que alcancen al finalizar el trabajo.

Tarea (figura 2): en este apartado se

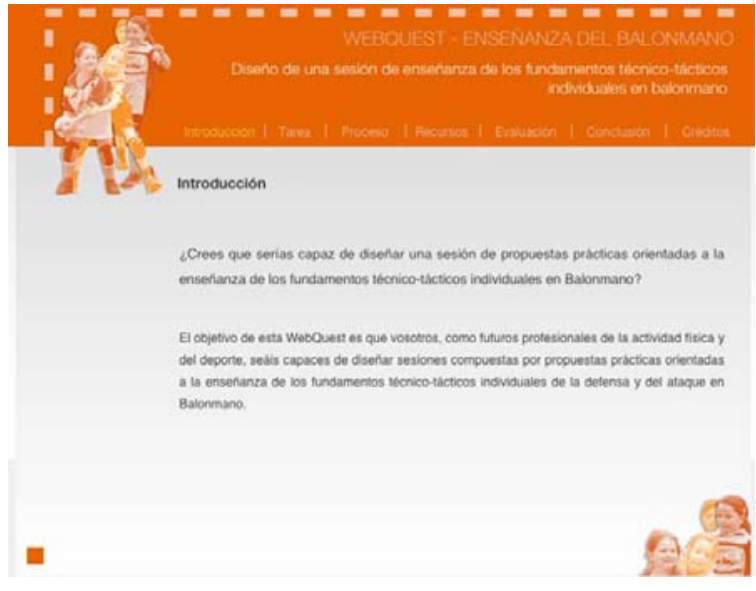

Figura 1. Introducción

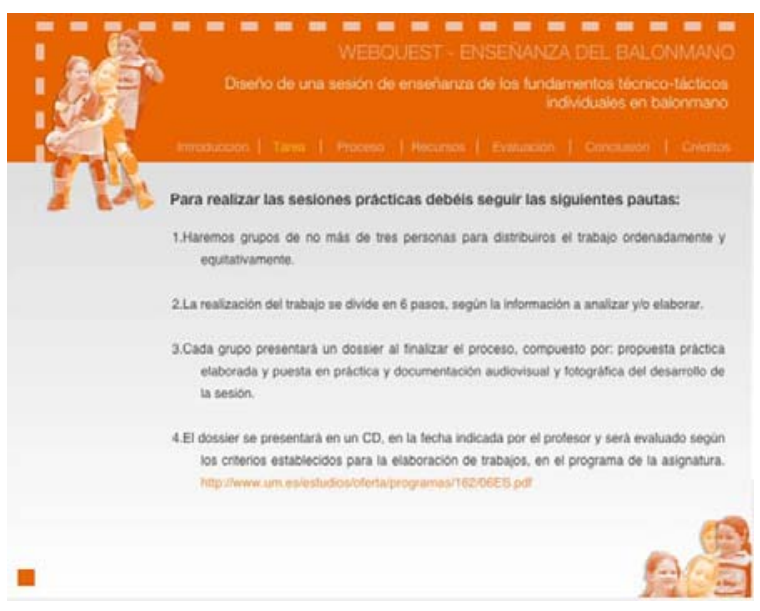

Figura 2. Tarea

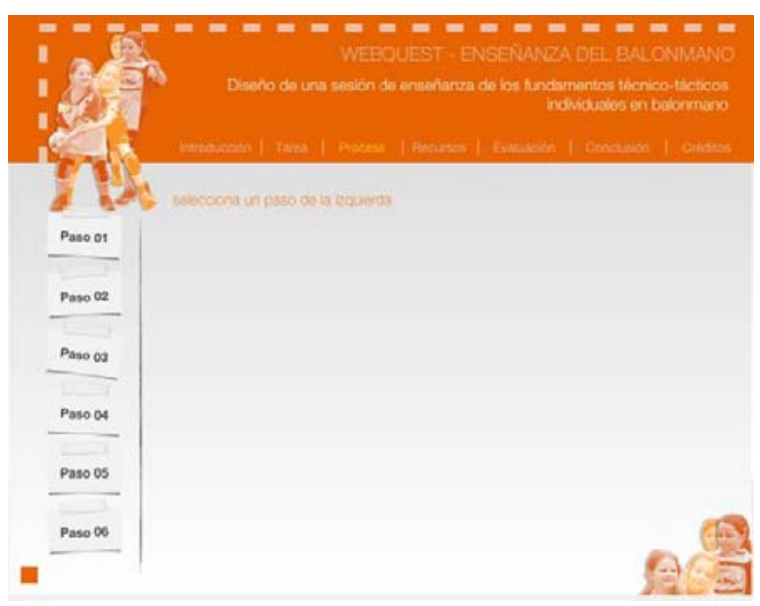

Figura 3. Proceso proporciona al alumno una descripción del producto final que tiene que elaborar a través de la WebQuest. Constituye un esquema guía que le ayudará a ver de forma global todos los pasos que deberá ir dando para finalizar con éxito la WebQuest. 
Procesos y recursos: en este apartado se sugieren los pasos que los alumnos deben seguir para completar la tarea. Su descripción debe ser breve y clara. Cada uno de los pasos incluirá los recursos que necesitan para ser llevados a cabo. Éstos pueden ser enlaces de Internet u otro tipo de documentos como libros, artículos, cuestionarios, etc. En nuestro caso, los pasos serían los siguientes (figura 3):

Paso 1: descripción de los objetivos del trabajo a realizar (figura 4)

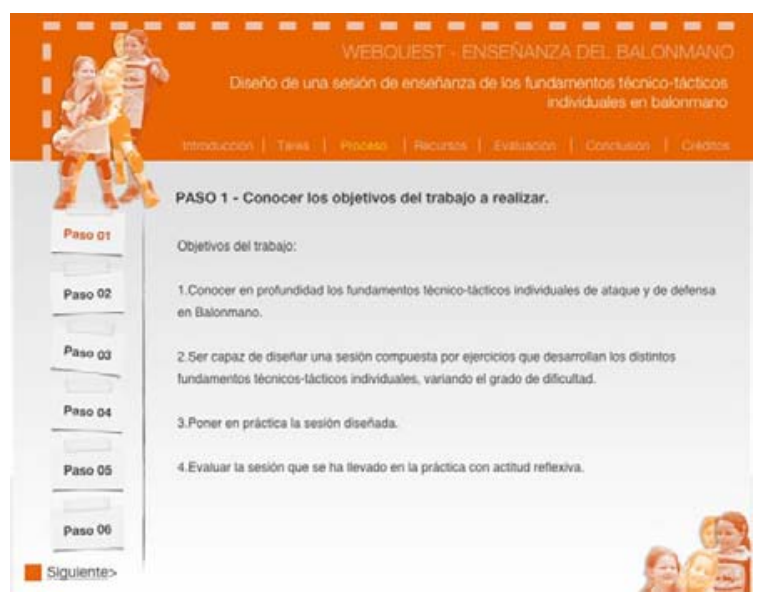

Figura 4. Paso 1

Paso 2: descripción de los contenidos a estudiar (figura 5)

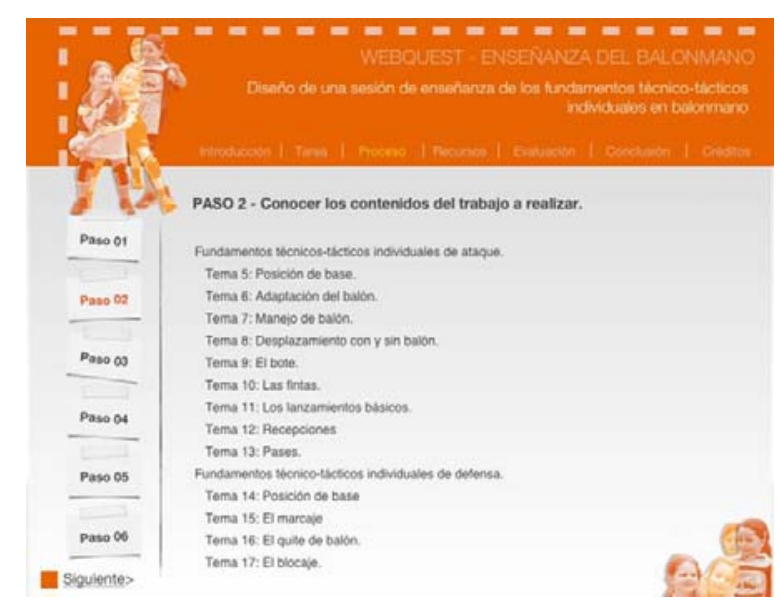

Figura 5. Paso 2

Paso 3: información de las referencias bibliográficas disponibles para desarrollar el trabajo (figura 6)

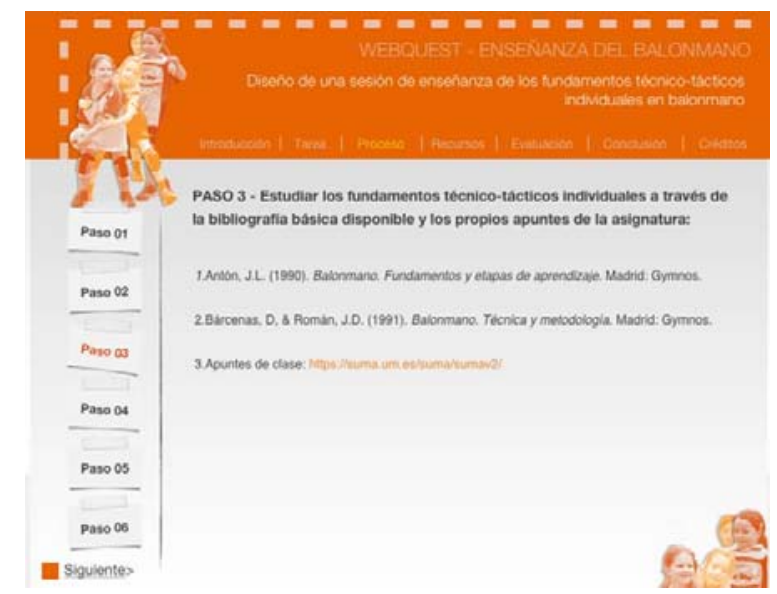

Figura 6. Paso 3 
$\checkmark$ Paso 4: explicación de cómo se diseña un guión de la sesión que tienen que realizar en sucio para presentarlo y debatirlo previamente a la exposición en tutoría (figuras 7 y 8).

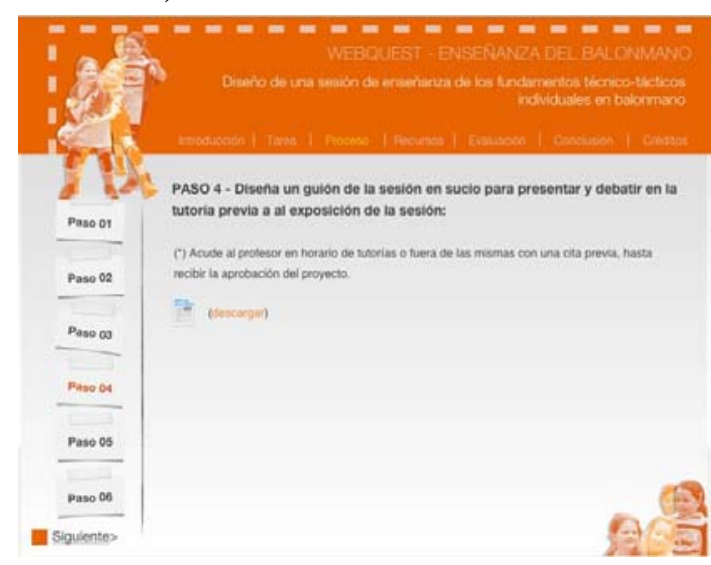

Figura 7. Paso 4

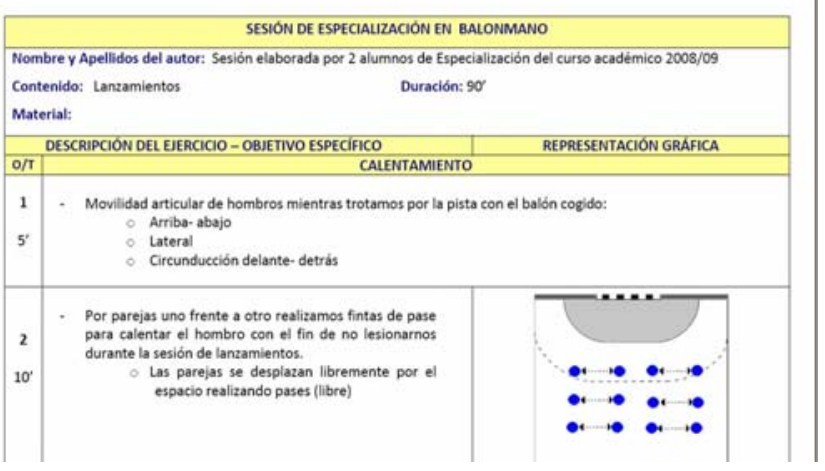

Figura 8. Ejemplo de sesión en pdf

$\checkmark \quad$ Paso 5: preparación de la presentación del trabajo en grupo (figura 9).

$\checkmark$ Paso 6: explicación de cómo y qué hay que entregar al profesor una vez terminada la experiencia (figura 10).

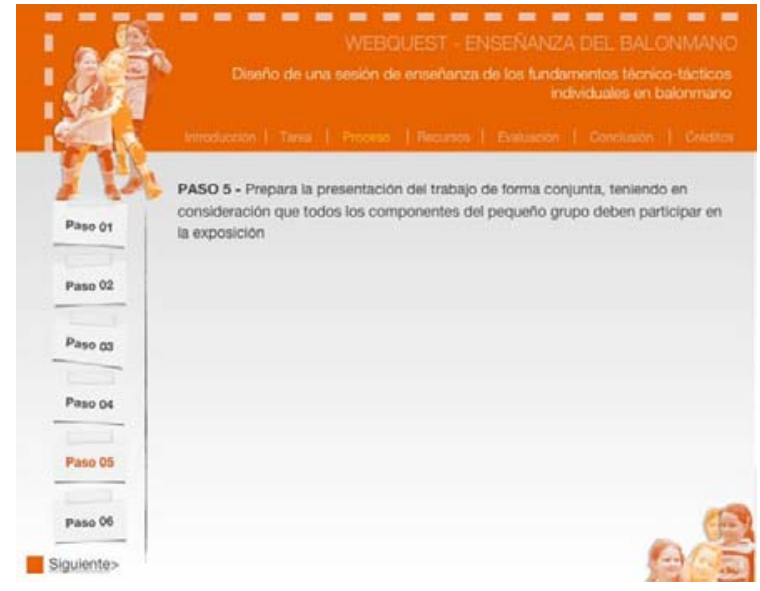

Figura 9. Paso 5

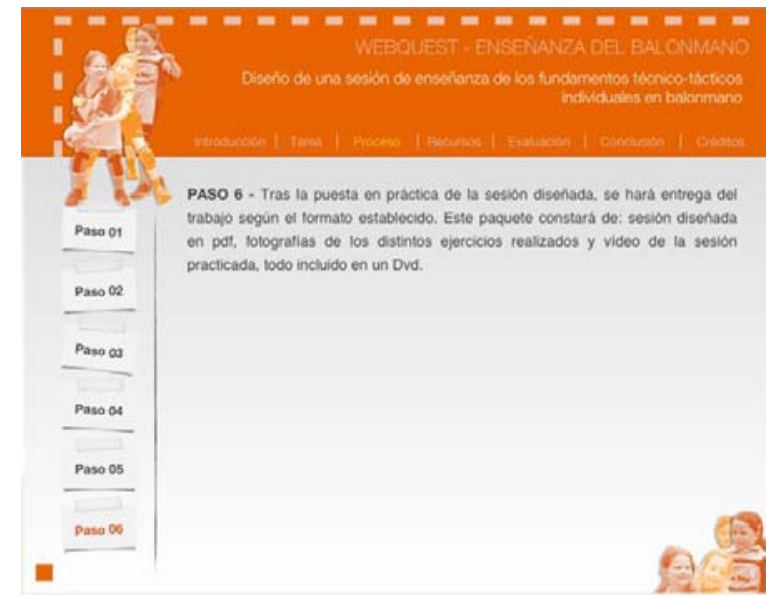

Figura 10. Paso 6

En cuanto al apartado de recursos, en el se le detallan una serie de links a otras páginas web relacionadas con el Balonmano, que son complementos de las referencias bibliográficas básicas a la hora de elaborar el trabajo (figura 11).

Evaluación (figura 12): aparece información sobre los ítems que el profesor utiliza para comprobar si los alumnos han adquirido los conocimientos. Los criterios evaluativos son precisos, claros, consistentes y específicos para el conjunto de tareas. Pero no sólo evalúa el profesor, es recomendable plantear una evaluación donde participen todos los implicados en el proceso de enseñanza-aprendizaje. En la evaluación profesor-alumno (figura 13) se le indican las puntuaciones que el profesor dará a cada uno de los subapartados. En la evaluación alumno-alumno (figura 14) se les pide a los alumnos que evalúen a cada uno de sus compañeros de grupo siguiendo unos criterios que tiene que valorar del 0 al 5 . Y por último se incluye una evaluación alumno-profesor (figura 15), en la que éste valorará el papel del profesor como guía en el desarrollo de su trabajo. 


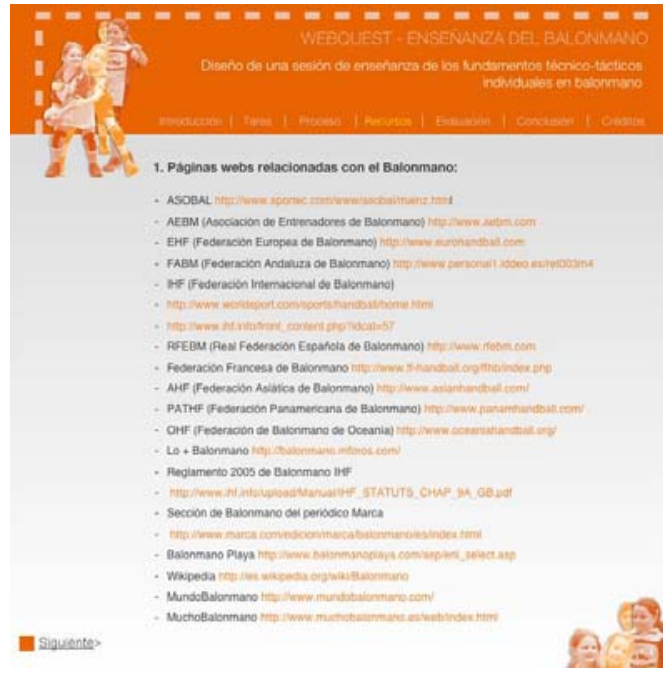

Figura 11. Recursos

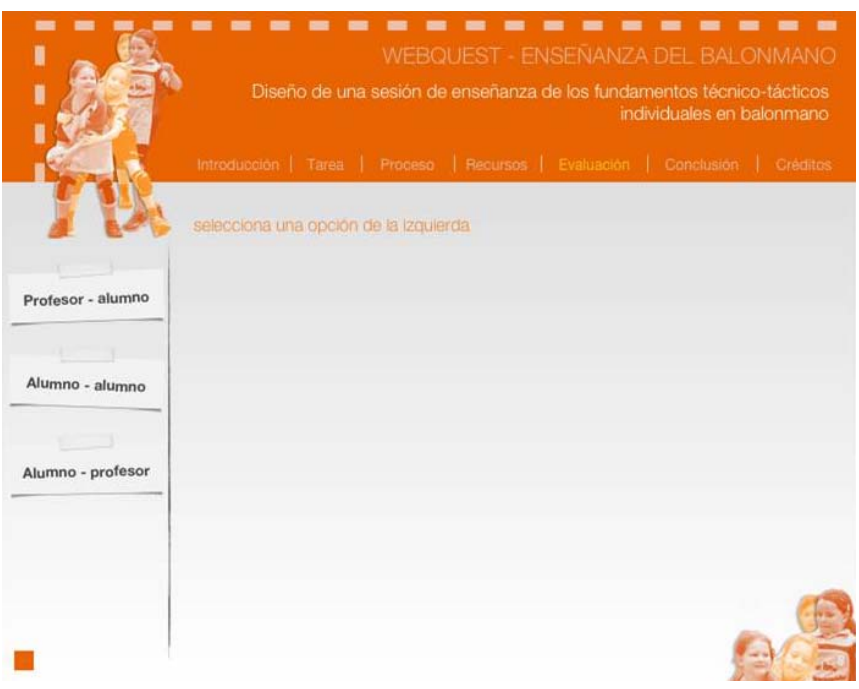

Figura 12. Evaluación

Conclusión: su finalidad es proporcionar la oportunidad de sintetizar la experiencia, animar a la reflexión sobre el proceso y generalizar lo que se ha aprendido. Puede ser interesante, en esta sección instar a los alumnos a aprender más sobre el tema o implicarlos en una investigación autónoma, darles nuevas pistas para poder continuar el trabajo de otras maneras: nuevas direcciones, enfoques, propuestas y creaciones (figura 16).

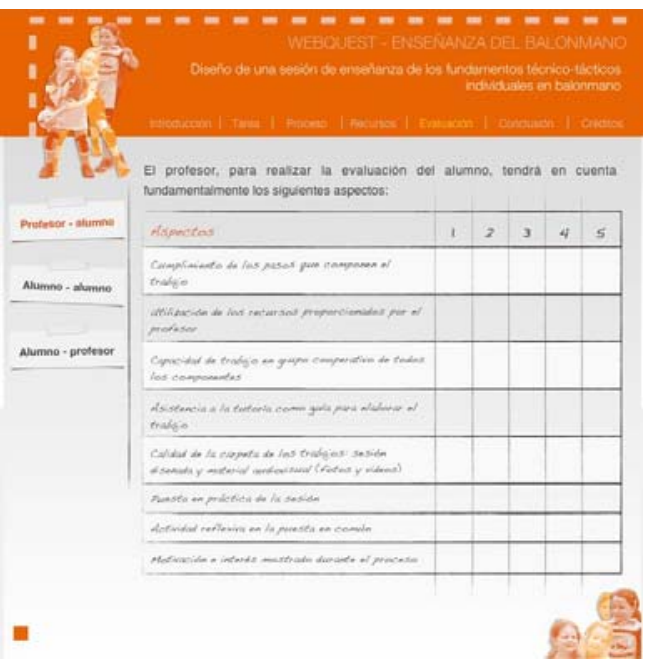

Figura 13. Profesor-alumno

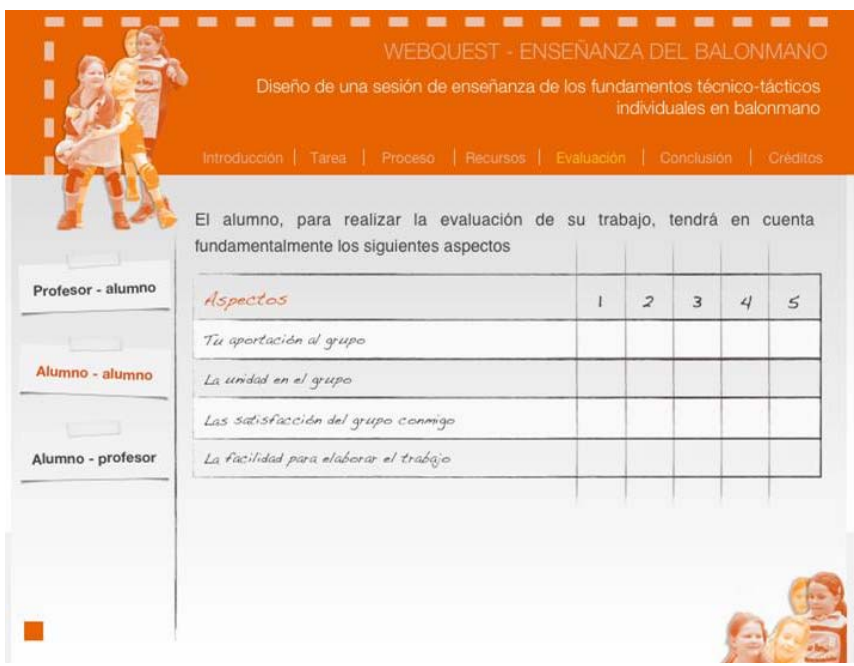

Figura 14. Alumno-alumno

\section{3.- CONCLUSIONES}

Con la propuesta que he mostrado en este trabajo he pretendido aportar un modelo docente alternativo de mayor calidad donde el alumnado sea el protagonista de su propio aprendizaje dentro del ámbito del deporte (Carrasco, Gómez-López, Abraldes y Ureña, 2011). Se ha podido comprobar cómo la WebQuest constituye una estrategia didáctica adecuada para la utilización de recursos tecnológicos en la docencia. Además, la presentación de contenidos, actividades de aprendizaje y tareas de autoevaluación a través de las TIC, posibilita una formación individualizada y un aprendizaje autorregulado del alumno, que facilita el incremento de su capacidad de gestión y autonomía en la planificación y adquisición de competencias profesionales. 
Resaltar también que el uso de las TIC impulsa el desarrollo de proyectos colaborativos entre el alumnado (Rodríguez y González, 2011) aunque tal y como señalan Echazarreta, Prados, Poch y Soler (2009), el éxito de los mismos dependerá de una adecuada planificación y estructuración previa por parte del profesor.

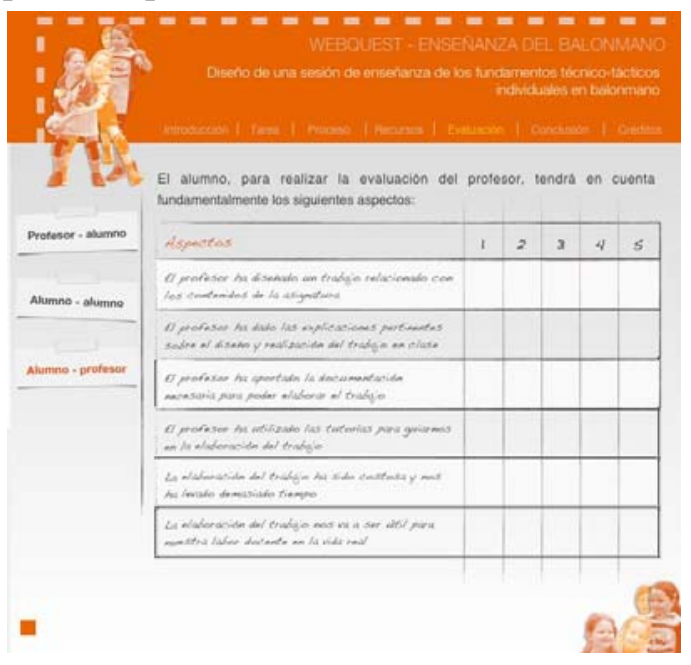

Figura 15. Alumno-profesor

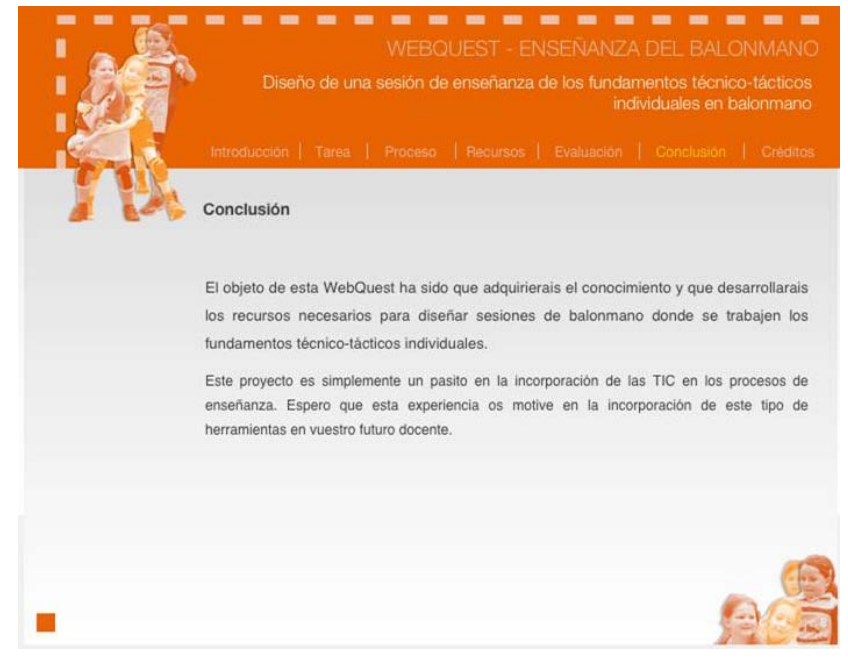

Figura 16. Conclusión

\section{4.- REFERENCIAS BIBLIOGRÁFICAS}

Adell, J. (2004). Internet en el aula: Las WebQuest. Edutec. Revista Electrónica de Tecnología Educativa, 17. http://edutec.rediris.es/Revelec2/revelec17/adell_16a.htm [Consulta: abril. 2012].

Area, M. (2004). WebQuest. Una estrategia de aprendizaje por descubrimiento basada en el uso de Internet. Quaderns Digitals,

33. http://www.quadernsdigitals.net/index.php?accionMenu=hemeroteca.VisualizaArticuloIU.visualiza\&art iculo_id=7374 [Consulta: abril. 2012].

Benito, M. y Ovelar, R. (2005). Impacto de las TIC y del proceso de convergencia al EEES en el profesorado universitario. PULSAR. http://pulsar.ehu.es/pulsar/documentacion/informes_pulsar/Informe_PULSAR_Diciembre.pdf [Consulta: abril. 2012].

Carrasco, M., Gómez-López, M., Abraldes, J. A., y Ureña, N. (2011). La WebQuest como estrategia didáctica. Una aplicación práctica en el ámbito de las Ciencias del Deporte. En Actas del I Congreso Internacional de Innovación Profesor. Murcia: Universidad de Murcia. Universidad Politécnica de Cartagena.

Castro, N. (2007). Las WebQuest como recurso didáctico en educación física. En Actas del II Congreso Internacional y XXIV Nacional de Educación Física. Islas Baleares: Universitat de les Illes Balears.

Díaz, J. (2005). Utilización didáctica de Internet en educación física. http://www.xtec.es/jdiaz124/cursoudi/curso2.html [Consulta: abril. 2012].

Dodge, B. (1995). Some thoughts about WebQuests http://edweb.sdsu.edu/courses/edtec596/about_WebQuest.html [Consulta: abril. 2012].

Echazarreta, C., Prados, F., Poch, J., y Soler, J. (2009). La competencia -El trabajo colaborativo-: una oportunidad para incorporar las TIC en la didáctica universitaria. Descripción de la experiencia con la $\begin{array}{llll}\text { plataforma } & \text { ACME } & \text { (UdG). }\end{array}$ http://www.uoc.edu/uocpapers/8/dt/esp/echazarreta_prados_poch_soler.pdf [Consulta: abril. 2012].

Fernández, J. P. (2007). La aplicación de las TICs en el área de Educación Física a través del modelo didáctico de la WebQuest. Revista Iberoamericana de Educación, 44(5), 1681-5653.

Huertas, J. M., y Tenorio, A. F. (2006). Nuevas tecnologías en la didáctica de la estadística: WebQuest. Rect@, 1(27). En Actas de las XIV Jornadas de ASEPUMA y II Encuentro Internacional. Sevilla: Universidad Pablo de Olavide http://www.uv.es/asepuma/XIV/comunica/113NUEVA.pdf [Consulta: abril. 2012].

Mendaña, C., y González, B. (2004). El papel de las WebQuest como herramienta para el aprendizaje del alumno en la nueva sociedad del conocimiento. En Actas Virtuales del III Simposio Virtual de Computación en 
la Educación http://www.somece.org.mx/virtual2004/ponencias/contenidos/CuervoCristina.htm [Consulta: abril. 2012].

Núñez, N. (2011). La WebQuest, el aula virtual y el desarrollo de competencias para la investigación. Revista Iberoamericana de Educación, 55(1). http://www.rieoei.org/deloslectores/3717Nunez.pdf [Consulta: abril. 2012].

Rodríguez, M., y González, M. (2011). El uso de las TIC en el nuevo contexto del Espacio Europeo de Educación Superior según los directivos de la UDC. En Actas del XII Congreso Internacional de Teoría de la Educación. Barcelona: Universitat de Barcelona $<$ http://www.cite2011.com/Comunicaciones/TIC/019.pdf> [Consulta: mayo. 2012].

Ruiz, J., Navarrete, C., Martínez, J. M., y González, D. (2006). Innovación y nuevas tecnologías en la Educación Universitaria: el proyecto ¿Quién se ha llevado mi queso? En Actas del V Congreso internacional “educación y sociedad”. Granada: Universidad de Granada.

San Miguel del Hoyo, B. (2011). Las tecnologías de la información y la comunicación en el Espacio Europeo de Educación Superior [Editorial]. Salud Colectiva, 7 (Supl 1), S5-S7.

Sosa, M. J. (2008). La WebQuest: ventajas e inconvenientes como recurso didáctico. En Actas del V Simposio Pluridisciplinar sobre Diseño y Evaluación de Contenidos Educativos Reutilizables. Salamanca: Universidad Pontificia de Salamanca. <http://www.web.upsa.es/spdece08/posters.html> [Consulta: mayo. 2012].

Starr, L. (2000). Meet Bernie Dodge -the Frank Lloyd Wright of Learning environments!. Education World http://www.education-world.com/a_tech/tech020.shtml [Consulta: mayo. 2012].

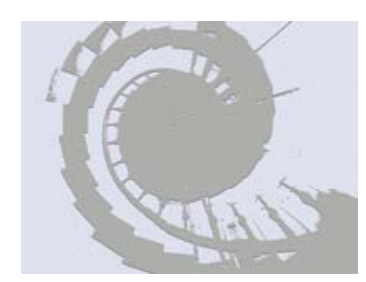

